https://doi.org/10.15407/ujpe64.1.11

F.A. MAJEED, F.A. MAHDI

Department of Physics, College of Education for Pure Sciences, University of Babylon (Hillah, Iraq; e-mail: fouadattia@gmail.com)

\title{
QUANTUM MECHANICAL CALCULATIONS OF A FUSION REACTION FOR SOME SELECTED HALO SYSTEMS
}

\section{Introduction}

The quantum-mechanical tunneling presented in halo nuclei produces unexpected effects. The energy needed to remove halo nucleons is drastically less than particle separation energies for typical nuclei. Nuclear radii are enhanced, but matter and charge radii may differ considerably. There is an evidence that few-body effects may become crucial, leading to the formation of cluster structures beyond the scope of mean-field theories. In the first approximation, the spatial separation of particles in the halo from the rest of the system justifies a simplified description with only a few active constituents [1]. Halo nuclei can be thought of in terms of a few (typically one or two) single halo nucleons orbiting a tightly bound core, thus implying a major role of single-particle properties. In quantitative terms, it has been assessed that, for a quantum halo to develop [2], first: the probability to find halo particles in the forbidden region beyond the classical turning point should be more than $50 \%$.

(C) F.A. MAJEED, F.A. MAHDI, 2019

ISSN 2071-0194. Ukr. J. Phys. 2019. Vol. 64, No. 1
The core-halo configuration should occur with more than the $50-\%$ probability in a given system. It has been argued $[3,4]$ that, for a nucleus to meet these criteria, $(a)$ : the energy needed to separate the halo part from the rest of the nucleus should be small, more precisely less than about $2 \mathrm{MeV} \cdot \mathrm{A}^{-2 / 3}$, with $A$ being the mass number of the nucleus; $(b)$ : the halo nucleons should occupy $s$ - or $p$-angular momentum orbits around the core; $(c)$ : the proton number of the nucleus should not exceed ten or so for a proton halo to develop. For three-body halo states containing two loosely bound nucleons, condition $b$ should be supplemented by the requirement for the hypermomentum $K$ to be 0 or 1 . The formation of a charged halo is hindered by the Coulomb barrier. The nucleus ${ }^{4} \mathrm{He}$ breaks up into ${ }^{3} \mathrm{He}+\mathrm{n}$ or ${ }^{4} \mathrm{He}(\mathrm{p}, \mathrm{pn})^{3} \mathrm{He}$ with the neutron separation energy $S_{n}=20.580 \mathrm{MeV} ;{ }^{6} \mathrm{Li}$ breaks up into ${ }^{4} \mathrm{He}+2 \mathrm{H}$, with the separation energy $S_{\alpha}=1.48 \mathrm{MeV}$, and ${ }^{7} \mathrm{Li}$ into ${ }^{4} \mathrm{H}+3 \mathrm{H}$, with $S_{\alpha}=2.45 \mathrm{MeV}[5,6]$. In order to consider the effect of the breakup channel for systems involving weakly bound projectiles, the coupling to the continuum was considered $[6,7]$. For practical pur- 
poses, it becomes necessary to approximate the continuum by a finite set of states, as in the Continuum Discretized Coupled-Channels method (CDCC) [8]. Recently, Majeed and Abdul-Hussien [9] had employed a semiclassical approach to study the effect of the breakup channel using the CDCC method on the fusion reaction cross-section $\sigma_{\text {fus }}$ and the fusion barrier distribution $D_{\text {fus }}$ for ${ }^{6,8} \mathrm{He}$ halo nuclei. Majeed et al. had performed semiclassical coupled-channels calculations in a heavy-ion fusion reaction for the systems ${ }^{40} \mathrm{Ar}+{ }^{110} \mathrm{Pd}$ and ${ }^{132} \mathrm{Sn}+{ }^{48} \mathrm{Ca}$. They proved that the semiclassical approach including the coupling between the elastic channel and the continuum turns out to be very successful in describing the total fusion reaction cross-section $\sigma_{\text {fus }}$ and the fusion barrier distribution $D_{\text {fus }}$ below and above the Coulomb barrier for medium and heavy systems [10]. The effect of the breakup channel on fusion reactions of weakly bound systems by means of a semiclassical and full quantum mechanical approaches has been discussed by F.A. Majeed [11]. Majeed et al. performed coupled-channel calculations using semiclassical and full quantum mechanical calculations to study the effect of channel coupling on the calculations of the total fusion reaction cross-section $\sigma_{\text {fus }}$, the fusion barrier distribution $D_{\text {fus }}$, and the fusion probability $P_{\text {fus }}$ for the light systems ${ }^{4} \mathrm{He}+{ }^{233} \mathrm{U},{ }^{13} \mathrm{C}+{ }^{48} \mathrm{Ti}$, and the medium system ${ }^{46} \mathrm{Ti}+{ }^{46} \mathrm{Ti}$. They argued that the inclusion of the coupled channels in their semiclassical and full quantum mechanical calculations enhances their calculations markedly below and around the Coulomb barrier $V_{b}$.

In this study, the effect of the breakup channel on the study of the fusion cross-section $\sigma_{\text {fus }}$, fusion barrier distribution $D_{\text {fus }}$, and mean angular momentum $\langle L\rangle$ for the systems ${ }^{6} \mathrm{He}+{ }^{64} \mathrm{Zn}$, ${ }^{6} \mathrm{He}+{ }^{209} \mathrm{Bi},{ }^{8} \mathrm{~B}+{ }^{58} \mathrm{Ni}$, and ${ }^{11} \mathrm{Be}+{ }^{238} \mathrm{U}$ will be explored. These systems involve light halo nuclei as projectiles, and the effect of the breakup channel should be significant.

\section{Differential Cross-Section}

When considering a spherically symmetric potential, the angular momentum of a system is a constant of motion. In this way, the wave function $\chi_{l, m}(r, \theta, \phi)$ can be factored into the radial, $u_{l}(r)$, and angular, $Y_{l}^{m}(\theta, \phi)$, parts [12],

$\chi_{l, m}(r, \theta, \phi)=u_{l}(r) Y_{l}^{m}(\theta, \phi)$ likewise, the Schrödinger equation can be separated into a radial and angular forms. The radial equation reads $[13]$

$$
-\frac{\hbar^{2}}{2 \mu} \frac{d^{2}}{d r^{2}} u_{l}(r)+\left[V(r) \frac{\hbar^{2}}{2 \mu} \frac{l(l+1)}{r^{2}}\right] u_{l}(r)=E u_{l}(r),
$$

where the term $\left(\hbar^{2} l(l+1) / 2 \mu r^{2}\right)$ represents the centrifugal barrier of a particle moving along an orbit with angular momentum $l$. The scattering process can be understood as the interaction of several partial wave functions with angular momentum with the central potential. Only those particles with the angular momentum relative to the target less than a maximum value will interact effectively with the target nucleus [12]. For the elastic scattering, the asymptotic limit leads to the following approximation of Eq. (1) [12]:

$\chi_{l, m}(r, \theta, \phi) \rightarrow e^{i \mathbf{k} \mathbf{r}}+f(\theta, \phi) \frac{e^{i \mathbf{k} \mathbf{r}}}{r}$,

where we assume that the particles do not have any intrinsic spins, so that the total angular momentum is just the orbital angular momentum. Adopting along the $z$-axis, it is possible to express the scattering amplitude of a nuclear potential as follows [13]:

$f(\theta, \phi)=\frac{1}{2 i k} \sum_{l}(2 l+1)\left[S_{l}-1\right] P_{l}(\cos \theta)$.

Here, $P_{l}(\cos \theta)$ is the Legendre polynomial, and $S_{l}$ represents the unitary scattering matrix which is expressed in terms of the reflection coefficients $\eta_{l}$ and the scattering phase shifts $\delta_{l}[13]$,

$S_{l}=\eta_{l} e^{2 i \delta_{l}}$.

The reflection coefficients $\eta_{l}$ represent the amplitude attenuation of the $l^{\text {th }}$ partial wave. For a real potential, no attenuation is expected, since $\eta_{l}=1$ (for complex potentials, $\eta_{l}<1$ ). The scattering phase shifts $\delta_{l}$ correspond to the angular shift experienced by the $l^{\text {th }}$ partial wave compared to an undisturbed wave. An attractive potential $(V(r)<0)$ leads to a positive phase shift, whereas a repulsive potential $(V(r)>0)$ produces a negative phase shift. In the absence of a nuclear potential, all phase shifts vanish [12]. The existence of an electromagnetic interaction between two 
interacting particles requires the adoption of an extra term for the wave function, which asymptotically takes the form [13]

$\chi_{\mathrm{C}}(K r) \rightarrow \frac{e^{(i \mathbf{k} \cdot \mathbf{r}-\eta \ln 2 k r)}}{r}$

with a phase shift relative to an undisturbed wave which depends logarithmically on the distance $r$, the Sommerfeld parameter $\eta$, taking the above correction into account, the wave function of Eq. (1) has the form [14]

$\chi(r, \theta, \phi) \rightarrow \chi_{\mathrm{C}}(r, \theta)+\chi_{N}(r, \theta, \phi) \rightarrow\left[f_{\mathrm{C}}(\theta)+\right.$

$\left.+f_{N}(\theta, \phi)\right] \frac{e^{(i \mathbf{k} \mathbf{r}-\eta \ln 2 k r)}}{r}$.

The nuclear interaction wave $\chi_{N}(r, \theta, \phi)$ and the amplitude $f_{N}(\theta, \phi)$ incorporate all possible interactions except for the electromagnetic ones. The Rutherford scattering amplitude can be written as [13]

$f_{\mathrm{C}}(\theta)=\frac{\eta}{2 k \sin ^{2}(\theta / 2)} e^{-i\left(\eta \ln \left(\sin ^{2}(\theta / 2)\right)+2 \delta_{l}\right)}$,

where the scattering amplitude of the nuclear interaction is given by [15],

$f(\theta, \phi)=\frac{1}{2 i k} \sum_{l}(2 l+1) e^{2 i \delta_{l}}\left[S_{l}-1\right] P_{l}(\cos \theta)$.

The Coulomb phase shift $\delta_{l}$, which contains the effect of the electromagnetic interaction on the scattering amplitude $f_{N}(\theta, \phi)$, is given by the expression [16],

$\delta_{l}=|\Gamma(l+1+i \eta)|$.

Considering both the effects of the electromagnetic and nuclear interactions, the total scattering amplitude of a projectile on a target nucleus $A$ is expressed in the form [16],

$\frac{d \sigma}{d \Omega}(\theta, \phi)=\left|f_{\mathrm{C}}(\theta)+f_{N}(\theta, \phi)\right|^{2}$.

The total fusion cross-section is given by

$\sigma_{\text {fus }}=\frac{\pi}{k^{2}} \sum_{l}(2 l+1) P_{l}$,

and the angular momentum $\langle L\rangle$ can be given as

$\langle L\rangle=\frac{\sum_{l} l(2 l+1) P_{l}}{\sum_{l}(2 l+1) P_{l}}$.

ISSN 2071-0194. Ukr. J. Phys. 2019. Vol. 64, No. 1

\section{Fusion Barrier Distribution}

The measurements of the fusion barrier distribution $D_{\text {fus }}$ represent a new stage in studies of the heavy ion fusion. The fusion barrier distribution analysis is a valuable tool to understand the mechanism of fusion of two heavy nuclei and the role of their internal degrees of freedom leading to the fusion. The fusion barrier distribution has been shown to be sensitive to the data related to the nuclear structure such as the nuclear shapes, multiple excitations, the anharmonicity of nuclear surface vibrations, etc. For this purpose, high precision measurements of the fusion crosssection data are required and have been reported for many systems [17]. It has been suggested recently that the application of a novel analysis technique allows this distribution of barriers to be extracted directly from fusion data $[18,19]$ without the imposition of arbitrary shapes and symmetries [19]. The probability $P(l, E)$ for the absorption of the $l^{\text {th }}$ partial wave is then given by the Hill-Wheeler formula $[20,21]$

$P(l, E)=\frac{1}{\left[1+\exp \left(\frac{2 \pi\left(V_{B}-E\right)}{\hbar \omega}\right)\right]}$.

The cross-section data were fitted by using the approximate Wong formula taken from $[7,17,22,23]$

$\sigma_{F}^{\mathrm{W}}=R_{B}^{2} \frac{\hbar \omega}{2 E} \ln \left[1+\exp \left(\frac{2 \pi\left(E-V_{B}\right)}{\hbar \omega}\right)\right]$.

Since the second derivative is proportional to the delta function (see Refs. [7, 17, 22, 23]), we get

$D_{F}=\pi R_{B}^{2} \frac{\hbar \omega}{2 \pi} \delta\left(E-V_{B}\right)$

The tunneling effect smears the Dirac delta function in Eq. (16). For Eq. (15), we take the second derivative of $\left(E \sigma_{F}\right)$ as proportional to the $s$-wave penetrability for a parabolic barrier [23, 24]

$D_{F}=\frac{d^{2}}{d E^{2}}\left[E \sigma_{F}(E)\right]=$

$=\pi R_{B}^{2} \frac{2 \pi}{\hbar \omega} \frac{e^{x}}{\left(1+e^{x}\right)^{2}}=\pi R_{B}^{2} \frac{d P(E)}{d E}$,

where $x=\left(-2 \pi\left(E-V_{B}\right)\right) / \hbar \omega$. For a completely classical system, $P(E)$ is 1above the barrier and zero below it; hence, the quantity $d P(E) / d E$ will be a 


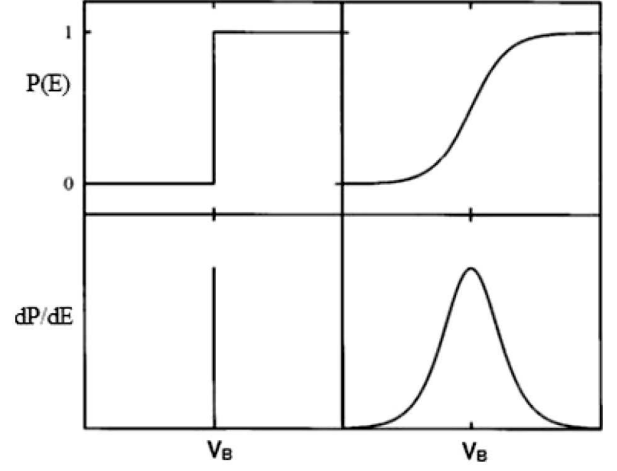

Fig. 1. Classical (on the left) and quantum-mechanical (on the right) transmission probabilities for a one-dimensional potential barrier [25]

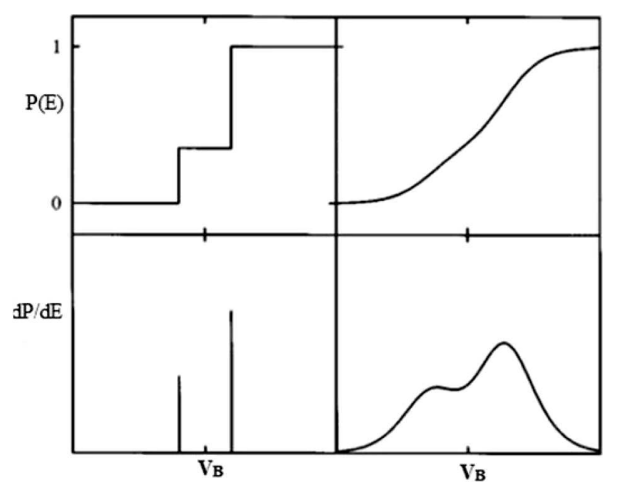

Fig. 2. Classical (on the left) and quantum-mechanical (on the right) transmission probabilities for a two-channel coupling. $V_{B}$ is the height of the one-dimensional potential barrier coupled to these channels [25]

Table 1. Parameters of the Aküz-Winther potential along with $V_{b}$

\begin{tabular}{|c|c|l|l|c|}
\hline Projectile + target & $V_{0}(\mathrm{MeV})$ & $a_{0}(\mathrm{fm})$ & $r_{0}(\mathrm{fm})$ & $V_{b}(\mathrm{MeV})$ \\
\hline${ }^{6} \mathrm{He}+{ }^{64} \mathrm{Zn}$ & -43 & 0.8 & 1.1 & 8.4 \\
${ }^{6} \mathrm{He}+{ }^{209} \mathrm{Bi}$ & -123.4 & 0.55 & 1.13 & 20.3 \\
${ }^{8} \mathrm{~B}+{ }^{58} \mathrm{Ni}$ & -86.2 & 0.803 & 1.098 & 19.75 \\
${ }^{11} \mathrm{Be}+{ }^{238} \mathrm{U}$ & -124.0 & 0.8 & 1.1 & 41.01 \\
\hline
\end{tabular}

delta function peaked, when $E$ is equal to the barrier height, as shown in Fig. 1 (left side). Quantum mechanically (see Fig. 1, right side), since the transmission probability smoothly changes from zero at energies far below the barrier to unity at energies far above the barrier, the sharp peak is broadened. It has been demonstrated [22] that this broadened peak presents a near Gaussian distribution with a width of $0.56 \hbar \omega$. In the case of the two-channel coupling, the quantity $d P(E) / d E$ is further broadening, owing to the overlapping of the two peaks, and it can be taken to represent the distribution of barriers, due to the coupling to extra degrees of freedom, as depicted in Fig. 2.

The presence of channel couplings immediately leads to [24]

$D_{F}(E)=\frac{d^{2}}{d E^{2}}\left[E \sigma_{F}(E)\right]=\sum_{\gamma} \frac{d^{2}}{d E^{2}}\left[E \sigma_{F}^{(\gamma)}(E)\right]$.

This function $D_{F}$ has been referred to as the fusion barrier distribution in units of $(\mathrm{mb} / \mathrm{MeV})$ and is related to the curvature $\frac{d^{2}}{d E^{2}}\left[E \sigma_{F}(E)\right]$, which has been extracted from data using the cross-sections $\sigma_{(F-)}, \sigma_{F}$, and $\sigma_{(F+)}$ at center-of-mass energies $E-$ $-\Delta E, E$, and $E+\Delta E$, respectively, by the threepoint difference formula $[22,26]$

$D_{F}(E)=\left\{(E-\Delta E) \sigma_{F-}-2 E \sigma_{F}+\right.$

$\left.+(E+\Delta E) \sigma_{F+}\right\} / \Delta E^{2}$.

The statistical uncertainty $\delta c$ associated with this curvature is given approximately by

$\delta c \cong\left(E / \Delta E^{2}\right)\left[\delta \sigma_{F-}^{2}+4 \delta \sigma_{F}^{2}+\delta \sigma_{F+}^{2}\right]^{1 / 2}$,

where the $\delta \sigma_{F}$ are random errors of the cross-sections.

\section{Results and Discussion}

In this section, the numerical results for the total fusion cross-section $\sigma_{\text {fus }}$, the fusion barrier distribution $D_{\text {fus }}$, and the mean angular momentum $\langle L\rangle$ for the systems ${ }^{6} \mathrm{He}+{ }^{64} \mathrm{Zn},{ }^{6} \mathrm{He}+{ }^{209} \mathrm{Bi},{ }^{8} \mathrm{~B}+{ }^{58} \mathrm{Ni}$, and ${ }^{11} \mathrm{Be}+{ }^{238} \mathrm{U}$ calculated within the full quantum mechanical approach are presented in Figs. 3, 4, 5, and 6 , respectively. Our calculated values of $\sigma_{\text {fus }}, D_{\text {fus }}$, and $\langle L\rangle$ are compared with the corresponding experimental data using the CC code. The Aküz-Winther potential parameters used in the present calculations are listed in Table 1. For all systems, our calculated results represented by dashed and solid red curves for the quantum calculations with coupled channels and without coupling, respectively.

ISSN 2071-0194. Ukr. J. Phys. 2019. Vol. 64, No. 1 

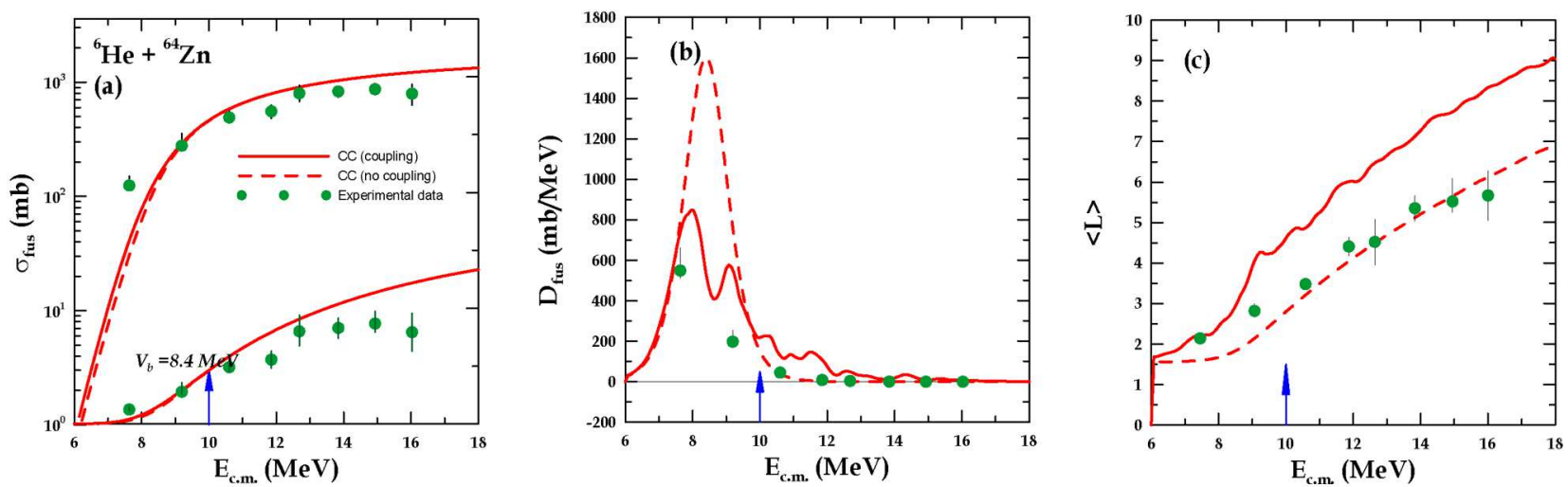

Fig. 3. The quantum mechanical calculations using $\mathrm{CC}$ code. The solid and dash lines are for the coupling and no coupling results, respectively, both compared with the experimental data (green filled circles) [27] for ${ }^{6} \mathrm{He}+{ }^{64} \mathrm{Zn}$ system. (a) for the total fusion cross-section $\sigma_{\text {fus }}(\mathrm{mb}),(b)$ for the fusion barrier distribution $D_{\text {fus }}(\mathrm{mb} / \mathrm{MeV})$, and $(c)$ for the mean value for the angular momentum $\langle L\rangle$. The blue arrow on the $E_{\text {c.m. }}$ axis represents the position of the Coulomb barrier $V_{b}$
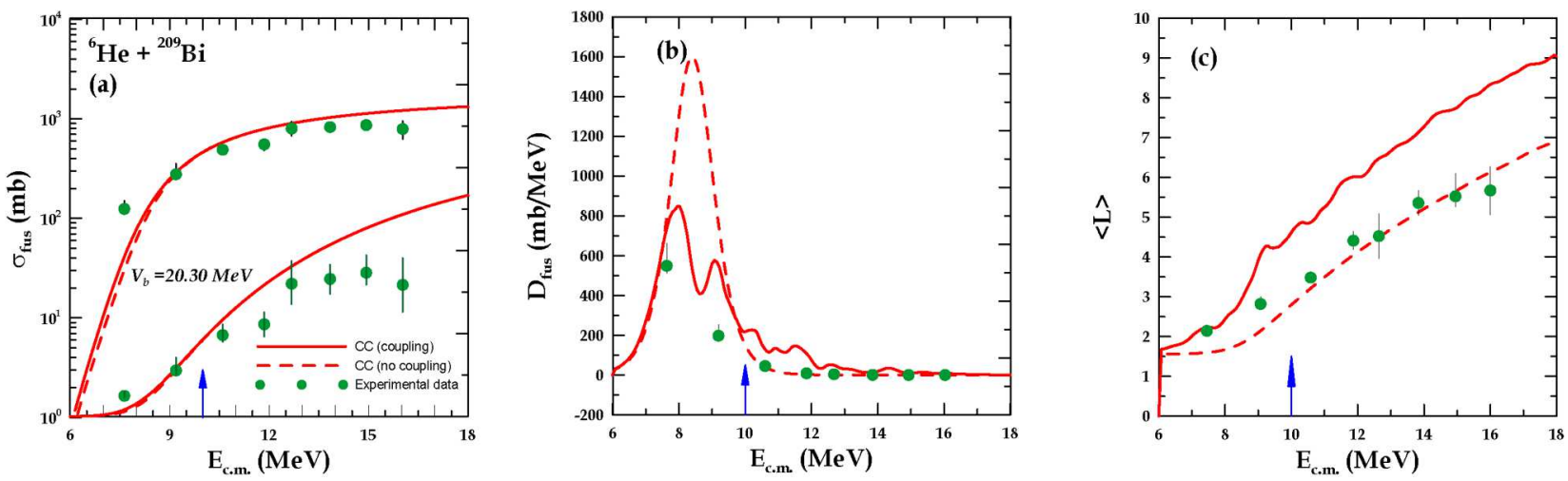

Fig. 4. The quantum mechanical calculations using $\mathrm{CC}$ code. The solid and dash lines are for the coupling and no coupling results, respectively, both compared with the experimental data (green filled circles) [28] for ${ }^{6} \mathrm{He}+{ }^{209} \mathrm{Bi}$ system. (a) for the total fusion cross-section $\sigma_{\text {fus }}(\mathrm{mb}),(b)$ for the fusion barrier distribution $D_{\text {fus }}(\mathrm{mb} / \mathrm{MeV})$, and $(c)$ for the mean value of the angular momentum $\langle L\rangle$. The blue arrow on $E_{\text {c.m. }}$. represents the position of the Coulomb barrier $V_{b}$

Figure $3, a, 3, b$ and $3, c$, respectively, presents the comparison between our results of the quantum approach calculations of the total fusion crosssection $\sigma_{\text {fus }}(\mathrm{mb})$, fusion barrier distribution $D_{\text {fus }}$ $(\mathrm{mb} / \mathrm{MeV})$, and mean angular momentum $\langle L\rangle$ with the corresponding experimental data for the system ${ }^{6} \mathrm{He}+{ }^{64} \mathrm{Zn}$. These results for coupled channels and without coupling are in good agreement with the experimental data above the Coulomb barrier $V_{b}$ for $\sigma_{\text {fus }}(\mathrm{mb})$ and $D_{\text {fus }}(\mathrm{mb} / \mathrm{MeV})$. The calculations and experimental data in all figures are plotted in both logarithmic and linear scales for the total fusion crosssection $\sigma_{\text {fus }}(\mathrm{mb})$. The inclusion of the coupling enhances the results of calculations for $\sigma_{\text {fus }}(\mathrm{mb})$ and $D_{\text {fus }}(\mathrm{mb} / \mathrm{MeV})$ below and above the Coulomb bar- rier $V_{b}$ indicated by a blue arrow located on the $E_{\text {c.m. }}$. axis. The calculations of the mean angular momentum including coupling effects overshoots the experimental data. Although the inclusion of the coupled channels makes the calculations of $D_{\text {fus }}$ and $\langle L\rangle$ in a better agreement with the experimental data, but it made the curves not smooth with ripples, which might be attributed to the sinusoidal behavior in the coupled differential equations, when the coupling is included. For this system, the mean angular momentum agrees well with the experimental data without coupling.

The comparison of our theoretical calculations of $\sigma_{\text {fus }}(\mathrm{mb}), D_{\text {fus }}(\mathrm{mb} / \mathrm{MeV})$, and $\langle L\rangle$ with the corresponding experimental data is shown in Figure 4, 

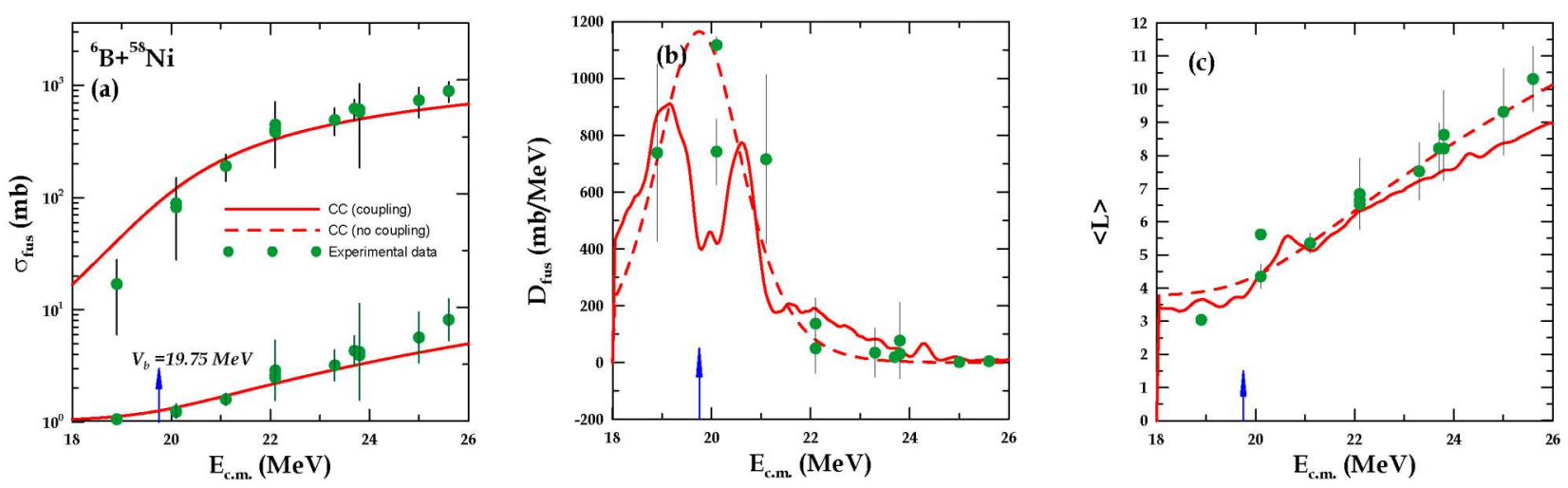

Fig. 5. The quantum mechanical calculations using $\mathrm{CC}$ code. The solid and dash lines are for the coupling and no coupling, respectively, both compared with the experimental data (green filled circles) [29] for ${ }^{8} \mathrm{~B}+{ }^{58} \mathrm{Ni}$ system. ( $a$ ) for the total fusion cross-section $\sigma_{\text {fus }}(\mathrm{mb}),(b)$ for the fusion barrier distribution $D_{\text {fus }}(\mathrm{mb} / \mathrm{MeV})$, and $(c)$ for the mean value for the mean angular

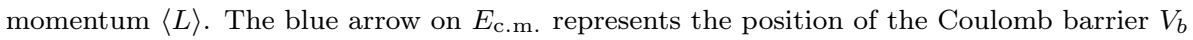
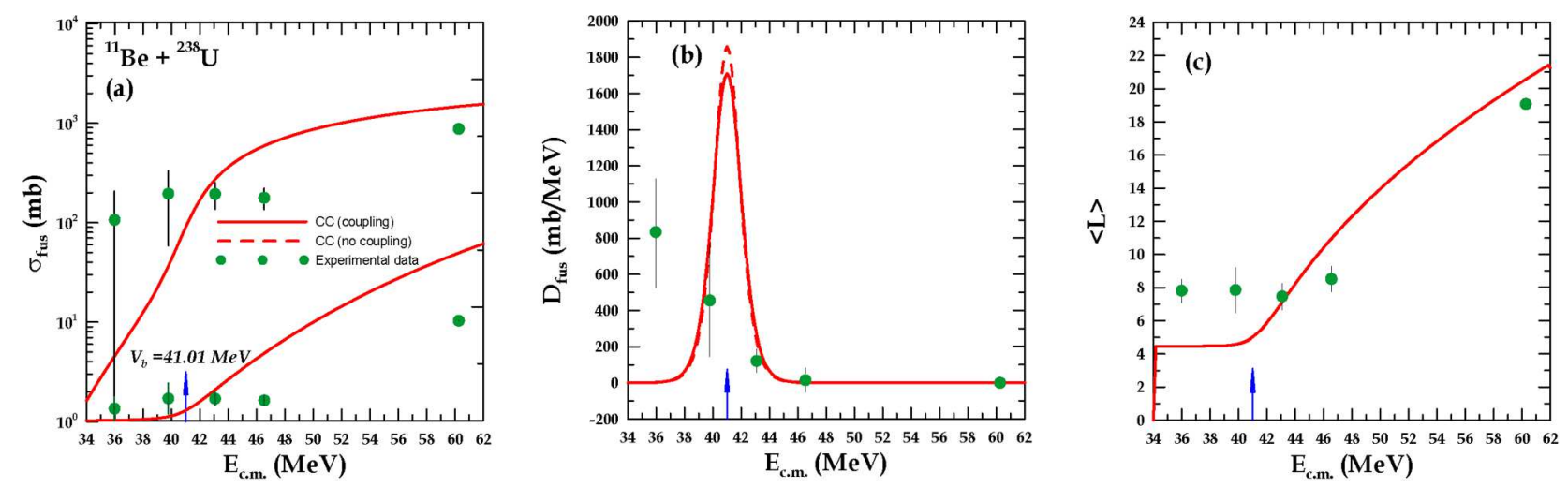

Fig. 6. The quantum mechanical calculations using CC code. The solid and dash lines are for the coupling and no coupling, respectively, both compared with the experimental data (green filled circles) [30] for ${ }^{11} \mathrm{Be}+{ }^{238} \mathrm{U}$ system. (a) for the total fusion cross-section $\sigma_{\text {fus }}(\mathrm{mb}),(b)$ for the fusion barrier distribution $D_{\text {fus }}(\mathrm{mb} / \mathrm{MeV})$, and $(c)$ for the mean value of the angular momentum $\langle L\rangle$. The blue arrow on $E_{\mathrm{c} . \mathrm{m}}$. represents the position of the Coulomb barrier $V_{b}$

panels $a, b$, and $c$, respectively, for the system ${ }^{6} \mathrm{He}+{ }^{209} \mathrm{~B}$. The inclusion of the channel coupling enhances the results of calculations of $\sigma_{\text {fus }}$ markedly below the Coulomb barrier $V_{b}$. For the fusion barrier distribution $D_{\text {fus }}$, the peak height is reduced with multiple peaks appeared instead of a single peak in the case of no coupling and becomes nearer to the experimental data. The inclusion of the channel coupling overestimates the experimental data for $\langle L\rangle$ above the Coulomb barrier $V_{b}$ and agrees very well below the Coulomb barrier $V_{b}$.

In a similar analysis, we compare our calculated results of $\sigma_{\text {fus }}, D_{\text {fus }}$, and $\langle L\rangle$ with the corresponding experimental data in panels $a, b$, and $c$ of Fig. 5, respectively, for the system ${ }^{8} \mathrm{~B}+{ }^{58} \mathrm{Ni}$. The experimental data of this system are obtained from Ref. [29]. The position of the experimental Coulomb barrier $V_{B}$ is indicated by an arrow at the $E_{\text {c.m. }}$ axis. The inclusion of the channel coupling seems have no effects on the calculations of $\sigma_{\text {fus }}$, while this is not the case for $D_{\text {fus }}$ and $\langle L\rangle$, which show a ripple behavior with two peaks appeared for $D_{\text {fus }}$, which are closer to the experimental data.

Figure 6 shows the comparison between our theoretical calculations of the total fusion cross-section $\sigma_{\text {fus }}$, fusion barrier distribution $D_{\text {fus }}$, and mean angular momentum $\langle L\rangle$ as displayed in panels $a, b$, and $c$, respectively, for the system ${ }^{11} \mathrm{Be}+{ }^{238} \mathrm{U}$. The experimental data for this system were obtained from Ref. [30]. For this system, the inclusion of coupled

ISSN 2071-0194. Ukr. J. Phys. 2019. Vol. 64, No. 1 
channels has almost no effect on the peak height of $D_{\text {fus }}$, shows same results in the case of no coupling, and, in general, has no agreement with the experimental data for $\sigma_{\text {fus }}$ and $\langle L\rangle$ except a very slight difference in the peak value of $D_{\text {fus }}$ exactly at the position of the Coulomb barrier $V_{b}$.

\section{Conclusion}

The quantum mechanical calculations for the total fusion reaction cross-section $\sigma_{\text {fus }}(\mathrm{mb})$, fusion reaction barrier distribution $D_{\text {fus }}(\mathrm{mb} / \mathrm{MeV})$, and mean angular momentum $\langle L\rangle$ have been performed for the systems ${ }^{6} \mathrm{He}+{ }^{64} \mathrm{Zn},{ }^{6} \mathrm{He}+{ }^{209} \mathrm{Bi},{ }^{8} \mathrm{~B}+{ }^{58} \mathrm{Ni}$, and ${ }^{11} \mathrm{Be}+{ }^{238} \mathrm{U}$. The effects of the coupled channels are found to be very important in the calculations and enhance the results of calculations markedly around and below the Coulomb barrier. The inclusion of the coupling effects for the full quantum mechanical calculations, by considering the coupling between the elastic and breakup channels, improves the results of calculations around and below the Coulomb barrier, but still has shortfall in reproducing the data, especially below the Coulomb barrier.

1. K. Riisager, D.V. Fedorov, A.S. Jensen. Quantum halos. Europhys. Lett. 49, 547 (2000).

2. A. Jensen, M. Zhukov. Few-body effects in nuclear halos. Nucl. Phys. A. 693, 411 (2001).

3. D. Fedorov, A. Jensen, K. Riisager. General properties of halos. Phys. Lett. B 312, 1 (1993).

4. A. Jensen, K. Riisager. Towards necessary and sufficient conditions for halo occurrence. Phys. Lett. B 480, 39 (2000).

5. A.W.K. Alder. Electromagnetic Excitations (North-Holland, 1975).

6. M. Dasgupta, D.J. Hinde, R.D. Butt, R.M. Anjos, A.C. Berriman, N. Carlin, P.R.S. Gomes, C.R. Morton, J.O. Newton, A. Szanto de Toledo, K. Hagino. Fusion versus breakup: Observation of large fusion suppression for ${ }^{9} \mathrm{Be}+{ }^{208}$ Pb. Phys. Rev. Lett. 82, 1395 (1999).

7. M. Dasgupta, D.J. Hinde, N. Rowley, A.M. Stefanini. Measuring barriers to fusion. Annu. Rev. Nucl. Part. Sci. 48, 401 (1998).

8. A. Diaz-Torres, I. J. Thompson, C. Beck. How does breakup influence the total fusion of ${ }^{6,7} \mathrm{Li}$ at the Coulomb barrier. Phys. Rev. C. 68, 044607 (2003).

9. F.A. Majeed, Y.A. Abdul-Hussien. Semiclassical treatment of fusion and breakup processes of ${ }^{6 ; 8} \mathrm{He}$ halo nuclei. J. Theor. Appl. Phys. 10, 107 (2016).

10. F.A. Majeed, R.S. Hamodi, F.M. Hussian. Effect of coupled channels on semiclassical and quantum mechanical calcu- lations for heavy ion fusion reactions. J. Comp. and Theor. Nanosci. 14, 2242 (2017).

11. F.A. Majeed. The role of the breakup channel on the fusion reaction of light and weakly bound nuclei. Int. J. Nucl. Energ. Sci. Tech. 11, 218 (2017).

12. A. Palumbo. $\alpha$-Capture and $\alpha$-Elastic Scattering on $P_{-}$ Nuclei to Probe the Hauser-Feshbach Framework. PhD thesis (Notre Dame Indiana, 2009).

13. S.S. Wong. Introductory Nuclear Physics (Wiley, 2008).

14. D. Ferry. Quantum Mechanics: An Introduction for Device Physicists and Electrical Engineers (Institute of Physics Publ., 2001).

15. D.J. Griffiths. Quantum Mechanics (Institute of Physics Publishing., 2001).

16. M.F.G. Auletta, G. Parisi. Quantum Mechanics (Cambridge University Press, 2009).

17. S.V.S. Sastry, S. Santra. Structure information from fusion barriers. Pra. J. Phys. 54, 813 (2000).

18. J.D. Bierman, P.Chan, J.F. Liang, M.P. Kelly, A.A. Sonzogni, R. Vandenbosch. Experimental fusion barrier distributions reflecting projectile octupole state coupling to prolate and oblate target nuclei. Phys. Rev. Lett. 76, 1587 (1996).

19. R.C. Lemmon, J.R. Leigh, J.X. Wei, C.R. Morton, D.J. Hinde, J.O. Newton, J.C. Mein, M. Dasgupta, N. Rowley. Strong dependence of sub-barrier fusion on the nuclear hexadecapole deformation. Phys. Lett. B 316, 32 (1993).

20. D.L. Hill, J.A. Wheeler. Nuclear constitution and the interpretation of fission phenomena. Phys. Rev. 89, 1102 (1953).

21. C.Y. Wong. Interaction barrier in charged-particle nuclear reactions. Rev. Lett. 31, 766 (1973).

22. N. Rowley, G. Satchler, P. Stelson. On the "distribution of barriers" interpretation of heavy-ion fusion. Phys. Lett. B 254, 25 (1991).

23. N. Takigawa, T. Masamoto, T. Takehiz, T. Rumin. Heavy ion fusion reactions and tunneling nuclear microscope. Jour. Kor. Phys. Soci. 43, 91 (2003).

24. K. Hagino, N. Takigawa. Subbarrier fusion reactions and many-particle quantum tunneling. Prog. Theor. Phys. 128, 1061 (2012).

25. A.B. Balantekin, N. Takigawa. Quantum tunneling in nuclear fusion. Rev. Mod. Phys. 70, 77 (1998).

26. J.X. Wei, J.R. Leigh, D.J. Hinde, J.O. Newton, R.C. Lemmon, S. Elfstrom, J.X. Chen, N. Rowley. Experimental determination of the fusion-barrier distribution for the ${ }^{154} \mathrm{Sm}+{ }^{16} \mathrm{O}$ reaction. Phys. Rev. Lett. 67, 3368 (1991).

27. V.A. Di Pietro, P. Figuera, M. Fisichella, F. Amorini, C. Angulo, G. Cardella, E. Casarejos, M. Lattuada, M. Milin, A. Musumarra, M. Papa, M.G. Pellegriti, R. Raabe, F. Rizzo, N. Skukan, D. Torresi, M. Zadro. Fusion and direct reactions for the system ${ }^{6} \mathrm{He}+{ }^{64} \mathrm{Zn}$ at and below the Coulomb barrier Phys. Rev. C 84, 064604 (2011).

28. J.J. Kolata, V. Guimarães, D. Peterson, P. Santi, R. White-Stevens, P.A. DeYoung, G.F. Peaslee, B. Hughey, 
B. Atalla, M. Kern, P.L. Jolivette, J.A. Zimmerman, M.Y. Lee, F.D. Becchetti, E.F. Aguilera, E. Martinez-Quiroz, J.D. Hinnefeld. Sub-barrier fusion of ${ }^{6} \mathrm{He}$ with ${ }^{209} \mathrm{Bi}$. Phys. Rev. Lett. 81, 4580 (1998).

29. E.F. Aguilera, P. Amador-Valenzuela, E. Martinez-Quiroz, J. Fernández-Arnáiz, J.J. Kolata, V. Guimarães. Abovebarrier fusion enhancement of proton-halo systems. Phys. Rev. C 93, 034613 (2016).

30. V. Fekou-Youmbi, J.L. Sida, N. Alamanos, F. Auger, D. Bazin, C. Borcea, C. Cabot, A. Cunsolo, A. Foti, A. Gillibert, A. Lépine, M. Lewitowicz, R. Liguori-Neto, W. Mittig, E. Pollacco, P. Roussel-Chomaz, C. Volant, Y.Y. Feng. Sub-Coulomb fusion with halo nuclei. Nucl. Phys. A 583, 811 (1995).

Received 04.06.18
Ф.А. Маджид, Ф.А. Махді

КВАНТОВО-МЕХАНІЧНИЙ РОЗРАХУНОК РЕАКЦІЇ СИНТЕЗУ ДЛЯ ДЕЯКИХ ГАЛО СИСТЕМ

$\mathrm{P}$ е $з$ ю м е

У рамках квантово-механічного підходу обговорюється вплив каналу розвалу на реакцію синтезу в слабкозв'язаних системах. Розраховані повний переріз реакції синтезу $\sigma_{\text {fus }}$, розподіл бар'єра синтезу $D_{\text {fus }}$ i середній кутовий момент $\langle L\rangle$ для систем ${ }^{6} \mathrm{He}+{ }^{64} \mathrm{Zn},{ }^{6} \mathrm{He}+{ }^{209} \mathrm{Bi},{ }^{8} \mathrm{~B}+{ }^{58} \mathrm{Ni} \mathrm{i}{ }^{11} \mathrm{Be}+{ }^{238} \mathrm{U}$. Знайдено, що облік каналу розвалу дуже істотний при розрахунку реакції синтезу в системах з легкими гало ядрами, особливо нижче кулонівського бар'єра $V_{b}$. Результати обчислень $\sigma_{\text {fus }}, D_{\text {fus }}$ i $\langle L\rangle$ добре узгоджуються з відповідними експериментальними даними. 